\title{
Risk factors for severe and critically ill COVID-19 patients: a review
}

\author{
Ya-dong Gao ${ }^{1}$, Mei Ding ${ }^{2}$, Xiang Dong ${ }^{2}$, Jin-jin Zhang ${ }^{2}$, Ahmet Kursat AZKUR ${ }^{3}$, Dilek \\ Azkur ${ }^{4}$, Hui Gan ${ }^{2}$, Yuan-li Sun ${ }^{2}$, Wei Fu ${ }^{2}$, Wei Li ${ }^{2}$, Hui-ling Liang ${ }^{2}$, Yi-yuan Cao ${ }^{5}$, Qi \\ $\mathrm{Yan}^{6}$, Can $\mathrm{Cao}^{2}$, Hong-yu Gao ${ }^{6}$, Marie-Charlotte Brüggen ${ }^{7}$, Willem van de Veen ${ }^{8}$, Milena \\ Sokolowska $^{9}$, Mubeccel Akdis ${ }^{10}$, and Cezmi Akdis ${ }^{8}$ \\ ${ }^{1}$ Zhongnan Hospital of Wuhan University \\ ${ }^{2}$ Wuhan University Zhongnan Hospital \\ ${ }^{3}$ Kirikkale University Faculty of Veterinary Medicine \\ ${ }^{4}$ Kirikkale Universitesi Tip Fakultesi \\ ${ }^{5}$ Wuhan University Zhongnan Hospital Department of Radiology \\ ${ }^{6}$ Tongji Hospital of Tongji Medical College of Huazhong University of Science and \\ Technology \\ ${ }^{7}$ University Hospital Zurich \\ ${ }^{8}$ University of Zurich \\ ${ }^{9}$ Swiss Institute of Allergy and Asthma Research, University of Zurich \\ ${ }^{10}$ University of Zürich
}

October 8, 2020

\begin{abstract}
The coronavirus disease 2019 pandemic (COVID-19), caused by the severe acute respiratory syndrome coronavirus 2 (SARS$\mathrm{CoV}-2$ ) has caused an unprecedented global social and economic impact, and numerous deaths. Many risk factors have been identified in the progression of COVID-19 into a severe and critical stage, including old age, male gender, underlying comorbidities such as hypertension, diabetes, obesity, chronic lung disease, heart, liver and kidney diseases, tumors, clinically apparent immunodeficiencies, local immunodeficiencies, such as early type-I interferon secretion capacity, and pregnancy. Possible complications include acute respiratory distress syndrome, shock, disseminated coagulopathy, acute kidney injury, pulmonary embolism, and secondary bacterial pneumonia. The development of lymphopenia and eosinopenia are laboratory indicators of COVID-19. Laboratory parameters to monitor disease progression include lactate dehydrogenase, procalcitonin, high-sensitivity C-reactive protein, proinflammatory cytokines such as interleukin (IL)-6, IL-1?, Krebs von den Lungen-6 (KL-6) and ferritin. The development of a cytokine storm and extensive chest computed tomography imaging patterns are indicators of a severe disease. In addition, socioeconomic status, diet, lifestyle, geographical differences, ethnicity, exposed viral load, day of initiation of treatment, and quality of health care have been reported to influence individual outcomes. In this review, we highlight the scientific evidence on the risk factors of COVID-19.
\end{abstract}

\section{Hosted file}

Risk factors for severe and critically ill COVID - final.pdf available at https://authorea. com/users/320601/articles/485583-risk-factors-for-severe-and-critically-ill-covid-19patients-a-review 
More comorbidities

Hormone difference
in inflammatory processes

Difference in levels

of ACE2, TMPRSS2

Lifestyle differences such as smoking

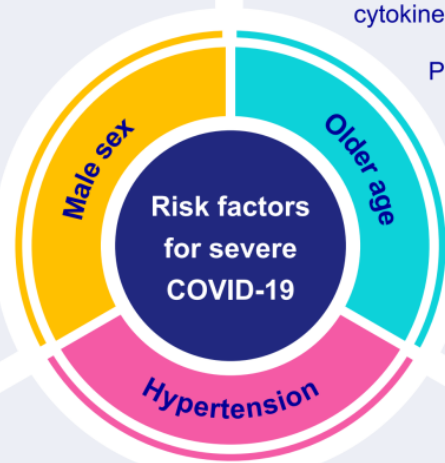

Weaker immune defense and higher levels of proinflammatory cytokines with increasing age

Possible reduced level of ACE2 in elderly

High SARS-CoV-2

viral load

Older age, and more complications and comorbidities, such as cardiovascular and cerebrovascular diseases, CKD and diabetes; imbalance between the 2 major RAAS pathways, ACE2/Ang-(1-7) and ACE/Ang ॥

Figure 1_Yadong et al. 
Detrimental restrictive ventilatory effect of abdominal fat

More complications and comorbidities

A prothrombotic condition

Immune dysregulation and chronic inflammation

Highly expressed

ACE2 in the epicardial

adipose tissue
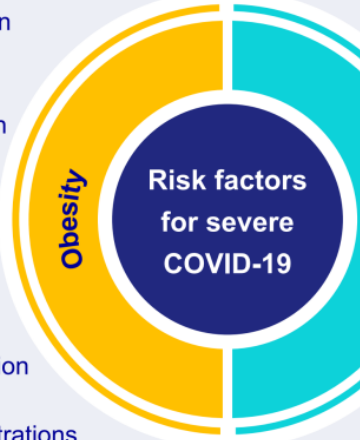

Higher prevalance of obesity

Liver steatosis

and abnormal liver function

Lower vitamin D concentrations

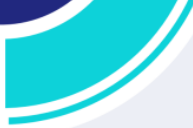

A prothrombotic state with an increased risk of thromboembolic events

Possible gut barrier leakiness

High SARS-CoV-2 viral load

Possible gut barrier leakiness

Figure 2_Yadong et al. 


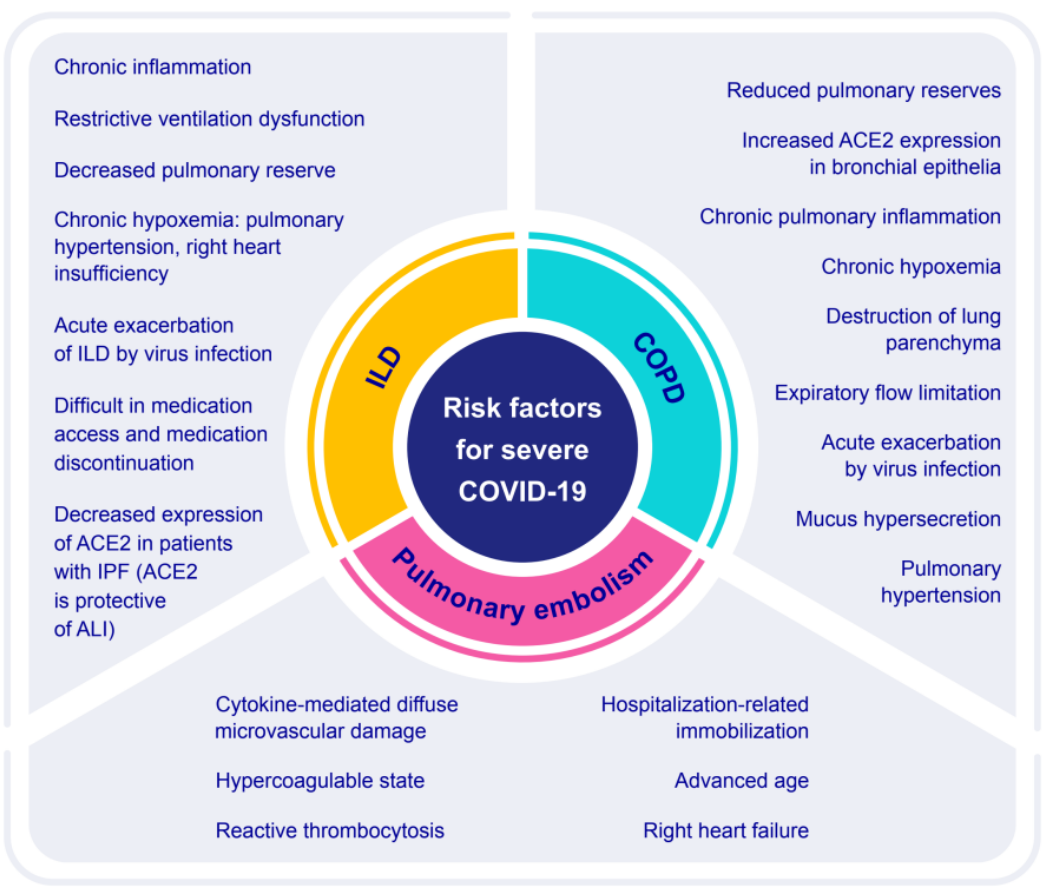

Figure 3_Yadong et al. 
Early robust IFN

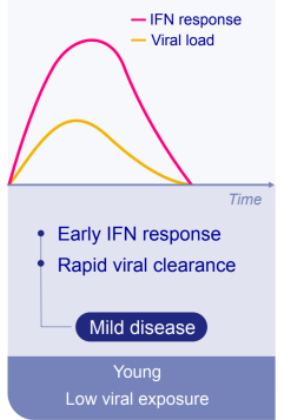

Delayed IFN

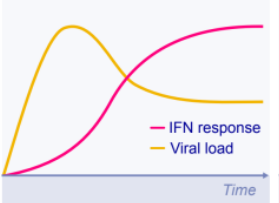

- Delayed IFN response

Viral persistence

Severe disease

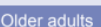

High viral exposure
IFN deficiency

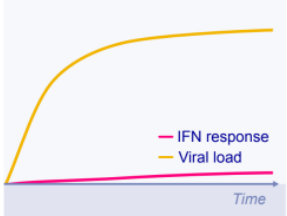

- Low or no IFN response

- No viral control,

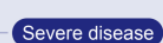

Genetic mutations in IFN pathways

Neutralizing Abs to IFNs
IFN therapy

- Recombinant IFN

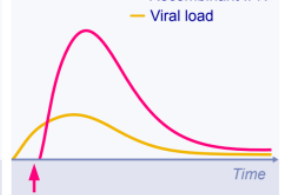

- Injection of recombinant IFN

- Viral clearance

Milder disease

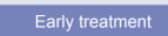

with recombinant IFN

Figure 4_Yadong et al. 


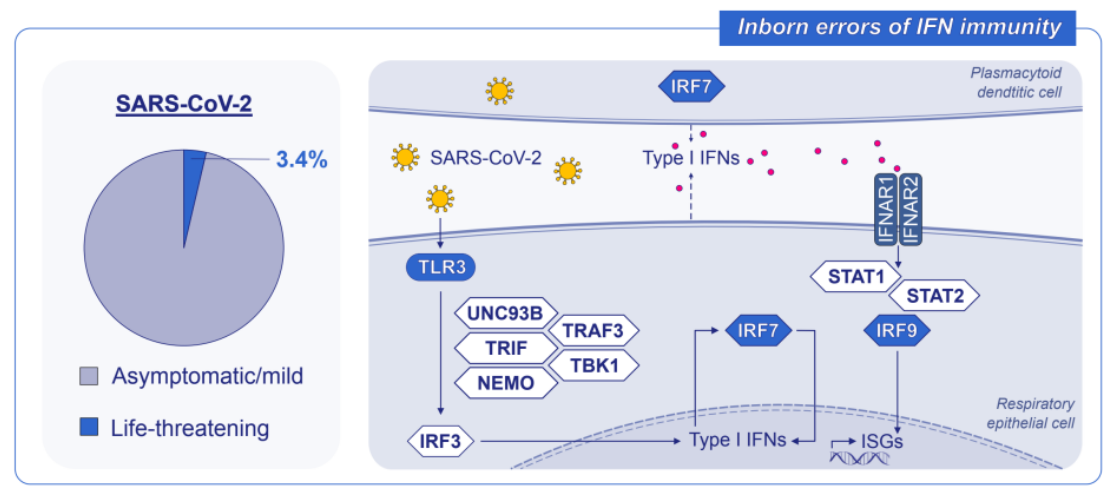

Figure 5_Yadong et al. 


\section{Laboratory indexes associated with severe and critical COVID}

\begin{tabular}{|c|c|c|c|c|c|}
\hline \multicolumn{2}{|c|}{$\begin{array}{l}\text { Peripheral blood } \\
\text { cell counts }\end{array}$} & \multicolumn{2}{|c|}{$\begin{array}{l}\text { Biochemical } \\
\text { parameters }\end{array}$} & \multicolumn{2}{|l|}{$\begin{array}{l}\text { Coagulation } \\
\text { indicators }\end{array}$} \\
\hline Leucocytes & $\uparrow$ & LDH & $\uparrow$ & Platelet counts & $\downarrow$ \\
\hline Lymphocytes & $\downarrow$ & CRP & $\uparrow$ & D-dimer & $\uparrow$ \\
\hline Neutrophils & $\uparrow$ & РCT & $\uparrow$ & Fibrinogen & $\uparrow$ \\
\hline Eosinophils & $\downarrow$ & AST/ALT & $\uparrow$ & PT & $\uparrow$ \\
\hline NLR & $\uparrow$ & BUN/Scr & $\uparrow$ & APTT & $\uparrow$ \\
\hline & & cTnl & $\uparrow$ & & \\
\hline & & IL-6 & $\uparrow$ & & \\
\hline & & IL-1 $\beta$ & $\boldsymbol{\uparrow}$ & & \\
\hline & & KL-6 & $\uparrow$ & & \\
\hline & & Ferritin & $\uparrow$ & & \\
\hline
\end{tabular}

Figure 6_Yadong et al. 
SARS-CoV-2

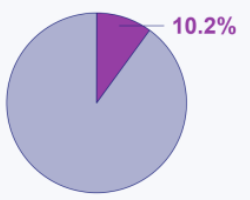

Asymptomatic/mild

Life-threatening
Type I IFN immunity
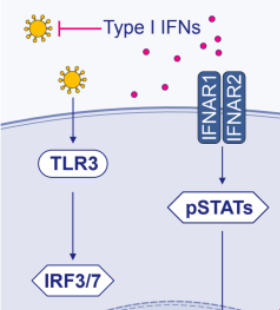

Auto-antibodies against type IIFN

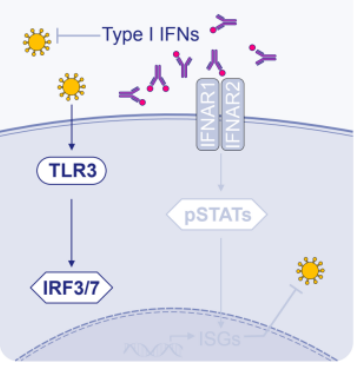

Figure 7_Yadong et al. 


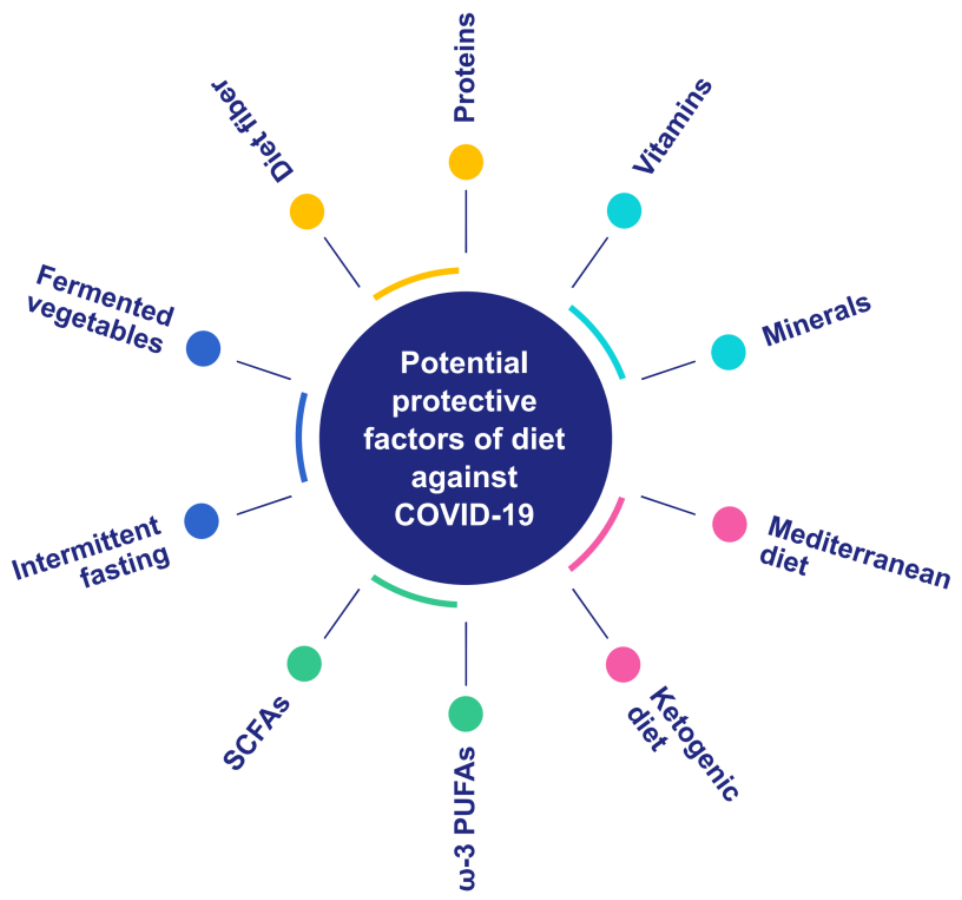

Figure 8_Yadong et al. 